Mens

Revue d'histoire intellectuelle et culturelle

Jacques Michon (dir.). Histoire de l'édition littéraire au Québec

au Xx ${ }^{e}$ siècle, t. 3 : La bataille du livre, 1960-2000, Montréal,

Fides, 2010, $520 \mathrm{p}$.

\title{
Simone Vannucci
}

Volume 12, numéro 1, automne 2011

URI : https://id.erudit.org/iderudit/1010573ar

DOI : https://doi.org/10.7202/1010573ar

Aller au sommaire du numéro

Éditeur(s)

Centre de recherche en civilisation canadienne-française

ISSN

1492-8647 (imprimé)

1927-9299 (numérique)

Découvrir la revue

Citer ce compte rendu

Vannucci, S. (2011). Compte rendu de [Jacques Michon (dir.). Histoire de

l'édition littéraire au Québec au xx ${ }^{e}$ siècle, t. 3 : La bataille du livre, 1960-2000,

Montréal, Fides, 2010, 520 p.] Mens, 12(1), 154-157.

https://doi.org/10.7202/1010573ar 
proprement canadienne-française, la naissance de la critique d'art et de revues spécialisées consacrées à la culture, la mise en place d'institutions d'enseignement des arts, la théorisation d'un art national et une brèche dans la liberté toute relative des artistes par rapport à leur création.

Bref, c'est avec éloquence et de nombreux détails que Vallée montre que, " par son engagement dans divers projets, activités et associations, Bourassa participe activement à la mise sur pied d'un réseau d'intellectuels partageant ses principaux idéaux » et qu'il a su faire la promotion de la culture canadienne-française, fondée sur un fort sentiment nationaliste et sur la tradition catholique, lui redonnant ainsi la juste place qui lui revient dans l'histoire de l'art du Québec.

- France St-Jean

Université d'Ottawa

\section{Jacques Michon (dir.). Histoire de l'édition littéraire au Québec au $X X^{e}$ siècle, t. 3 : La bataille du livre, 1960-2000, Montréal, Fides, 2010, 520 p.}

L'Histoire de l'édition littéraire au Québec au XX' siècle, t. 3 : La bataille du livre, 1960-2000 vient de paraître chez Fides. Cet ouvrage collectif publié sous la direction de Jacques Michon, professeur à l'Université de Sherbrooke, directeur du Groupe de recherche sur l'édition littéraire au Québec (GRELQ) et titulaire de la Chaire de recherche du Canada en histoire du livre et de l'édition, réunit des textes de Mélanie Beauchemin, Frédéric Brisson, Julie Frédette, Pierre Hébert, Marie-Pier Luneau, Suzanne Pouliot, Nathalie Watteyne avec l'assistance de René Davignon.

L'Histoire de l'édition littéraire au Québec montre, entre autres, comment les éditeurs du Québec, en plaçant le livre québécois au centre des enjeux sociaux, économiques, politiques et culturels, parviennent, au cours des années 1960 et des décennies suivantes, à propager les idées libérales, et comment, de ce fait, ils ont contribué à faire passer la pensée québécoise du XIX ${ }^{\mathrm{e}}$ siècle $\mathrm{au} \mathrm{Xx} \mathrm{X}^{\mathrm{e}}$. C'est là une des grandes qualités de ce tome III. 
À la mort de Duplessis, les professionnels du livre désirant faire reconnaître leurs droits se lancent dans une lutte destinée à mettre à bas les structures en place depuis presque un siècle et à contrer l'envahissement des grandes sociétés étrangères qui, depuis la fin de la guerre, tentaient d'occuper tout le champ de l'édition. Ils reprennent le combat de Robert Charbonneau qui, en 1947, dans son opuscule La France et nous, réclamait une aide gouvernementale pour mettre en place une édition nationale qui faciliterait l'essor de la littérature canadienne. Cette revendication restée sans écho tout au long de la décennie 1950 est reprise dès 1960 par le Conseil supérieur du livre (CSL). Après de nombreuses années de revendications, les éditeurs obtiendront gain de cause au début des années 1980 lorsque les deux paliers de gouvernement mettent sur pied des programmes d'aide à l'industrie du livre, ce qui permet l'envol de la production québécoise, comme le mentionne Jacques Michon dans l'introduction.

Les chapitres I à VI traitent, entre autres, du rôle joué par les éditeurs dans l'évolution de la pensée au cours des années 1960, qui se traduira notamment par l'introduction dans la société québécoise de nouvelles valeurs et de normes littéraires modernes. Cette évolution éditoriale est amorcée par Jacques Hébert avec les Éditions de l'Homme (1958) et les Éditions du Jour (1961), qui mettent en marché le livre à un dollar. Peu cher, vite imprimé sur papier journal et distribué dans les kiosques à journaux, il permet aux idées progressistes et laïques de la Révolution tranquille de s'implanter durablement. Ces publications, peu onéreuses et facilement accessibles à tous les publics, ouvrent la voie à la publication de toutes sortes d'ouvrages, dont l'un des plus célèbres, Les insolences du Frère Untel de Jean-Paul Desbiens, atteindra en quelques mois les 100000 exemplaires vendus.

Avec les Éditions du Jour qui appliquent la même formule que les Éditions de l'Homme, Jacques Hébert aspire à faire connaître les jeunes écrivains de la Révolution tranquille. Comme exemple, on peut citer le troisième roman de Marie-Claire Blais, Le jour est noir, qui dépasse les 6000 exemplaires vendus dans les mois qui suivent sa sortie. 
Les maisons fondées par Jacques Hébert font rapidement des émules. Les Éditions Parti pris, créées en 1965 par les jeunes directeurs de la revue du même nom, publient sous la direction de Gérald Godin des ouvrages politiques, sociologiques et philosophiques qui font quelques bruits. Pensons à Nègres blancs d'Amérique de Pierre Vallières publié en 1968, qui vaudra à Godin et Vallières d'être accusés de sédition. L'accusation sera abandonnée faute de preuves. Cependant, ces tribulations judiciaires, qui coûtent cher à la maison d'édition, provoquent sa fermeture. D'autres maisons plus modestes voient le jour et suivent le courant créé par Hébert.

Ce courant qui révolutionne la pensée québécoise au cours des années 1960 force les éditeurs déjà en place à des remises en question douloureuses sur le plan administratif et éditorial, qui entraînent des changements de cap et des mutations importantes. Mais si les tirages diminuent, ce qui est un phénomène mondial, on assiste au Québec à la plus forte croissance du nombre de titres publiés depuis les quarante dernières années. De 1971 à 1975, cette croissance permet à l'édition québécoise de faire reculer de façon significative la vente des livres étrangers, omniprésents sur le marché du Québec. De plus, de nouvelles activités, telles la traduction en français de best-sellers ou l'exportation du livre québécois, ouvrent de nouvelles voies commerciales aux éditeurs.

L'édition québécoise trouvera son point de stabilité au cours des années 1980, après plusieurs décennies de crise scrutées par de nombreuses commissions d'enquête. Cette stabilité proviendra essentiellement de la Loi sur le développement des entreprises québécoises dans le domaine du livre, promulguée en 1981, qui a favorisé notamment l'essor de la librairie.

Un autre des résultats importants induits par les transformations éditoriales de la dernière moitié du $\mathrm{XX}^{\mathrm{e}}$ siècle, soit les transformations de la censure, est traité par Pierre Hébert au chapitre XI du troisième volet de l'ouvrage. Sous le titre "Mutations de la censure ", il montre comment la disparition de la revue Lecture, de Fides, en 1966 et l'abolition de l'Index romain, notamment, affaiblissent considéra- 
blement la censure cléricale, laissant la place à une censure à caractère judiciaire qui vient réglementer les mœurs populaires et qui se substitue à la critique et aux interdits religieux.

Il arrivera cependant que les deux instances - censures cléricale et judiciaire - se soutiennent, notamment dans le scandale suscité par la présentation, en 1978, de la pièce de Denise Boucher Les fées ont soif. À l'approche du Xxi ${ }^{\mathrm{e}}$ siècle, les censures cléricale et judiciaire finissent par ne plus se pratiquer, laissant la place à une nouvelle forme de critique exercée par des groupes de pression : la censure d'obligation, plus difficile à reconnaitre que la censure d'interdiction, selon la démonstration de Pierre Hébert.

Le dernier tome de l'Histoire de l'édition littéraire au Québec au $X X^{e}$ siècle montre comment, après un demi-siècle de crises de toutes sortes, le système éditorial du Québec a, à partir des années 1980, trouvé un équilibre lui permettant de devenir concurrentiel par rapport aux maisons d'édition étrangères qui avaient investi l'espace éditorial. Ce changement est dû en partie à la loi de 1981 sur le développement des entreprises québécoises dans le domaine du livre, qui a favorisé la croissance de la librairie sur tout le territoire. Cette intervention de l'État dans le domaine culturel a été déterminante, car elle a permis de maintenir un équilibre entre petites et grandes structures, entre édition littéraire et édition purement industrielle, et a aidé les éditeurs à conquérir de nouveaux territoires à l'étranger.

Nous ne devons cependant pas oublier que c'est grâce au travail de pionniers tels que Robert Charbonneau ou Jacques Hébert que s'est développée l'édition québécoise. Ils ont publié à bas prix des œuvres et des auteurs dont les idées ont permis à la population du Québec, à la fin des années 1950 et au début de la décennie 1960, d'accueillir la Révolution tranquille et son corollaire, l'autonomie culturelle.

- Simone Vannucci Université de Sherbrooke 